\title{
Oxidative Phosphorylation Impairment by DDT and DDE
}

\author{
Sarah E. Elmore and Michele A. La Merrill* \\ Department of Environmental Toxicology, University of California, Davis, Davis, CA, United States
}

There is increasing evidence supporting the characterization of the pesticide DDT and its metabolite, DDE, as obesogens and metabolic disruptors. Elucidating the mechanism is critical to understanding whether the association of DDT and DDE with obesity and diabetes is in fact causal. One area of research investigating the etiology of metabolic diseases is mitochondrial toxicity. Several studies have found associations between mitochondrial defects and insulin resistance, cellular respiration, substrate utilization, and energy expenditure. Although the mitotoxicity of DDT and DDE was established 20-40 years ago, it was not viewed in the light of the diseases faced today; therefore, it is prudent

OPEN ACCESS

Edited by:

Robert Sargis,

University of Illinois at Chicago,

United States

Reviewed by:

Bradley Lane Baumgarner, University of South Carolina Upstate,

United States

Barton Wicksteed,

University of Illinois at Chicago,

United States

*Correspondence:

Michele A. La Merrill

mlamerrill@ucdavis.edu

Specialty section:

This article was submitted to

Systems and Translational

Endocrinology,

a section of the journal

Frontiers in Endocrinology

Received: 04 October 2018

Accepted: 11 February 2019

Published: 12 March 2019

Citation:

Elmore SE and La Merrill MA (2019) Oxidative Phosphorylation Impairment by $D D T$ and $D D E$.

Front. Endocrinol. 10:122.

doi: 10.3389/fendo.2019.00122 to reexamine the mitotoxicity literature for mechanistic support of DDT and DDE as causal contributors to obesity and diabetes, as well as associated diseases, such as cancer and Alzheimer's disease. This review aims to focus on studies investigating the effect of DDT or DDE on mammalian mitochondrial oxidative phosphorylation. We illustrate that both DDT and DDE impair the electron transport chain (ETC) and oxidative phosphorylation. We conclude that there is reasonable data to suggest that DDT and DDE target specific complexes and processes within the mitochondria, and that these insults could in turn contribute to the role of DDT and DDE in mitochondria-associated diseases.

Keywords: mitotoxicity, electron transport chain, insulin resistance, obesity, DDT, DDE, pesticides

\section{INTRODUCTION}

The discovery of dichlorodiphenyltrichloroethane (DDT) as an efficient insecticide won Paul Muller the Nobel Prize in the 1940s. Initially used to control vector-borne diseases, such as malaria, its broad use as a pesticide quickly grew. However, DDT was banned in 1972 in the United States due to adverse environmental effects. Although banned by many countries following the 2001 Stockholm Convention, DDT is still recommended for indoor residual spraying to control malaria vectors by the World Health Organization (1) and as such, continues to be manufactured and used. Current US FDA guidelines limit DDT and DDE levels to $0.05-5$ ppm depending on the commodity (2).

DDT and its metabolite dichlorodiphenyldicholorethylene (DDE) are both persistent organic pollutants (POPs) due to their physiochemical properties, allowing for biomagnification and their storage in the lipid-rich adipose tissue of mammals $(3,4)$. The environmental persistence of DDT and DDE, combined with the fact that DDT is still manufactured and used in parts of the world today, make DDT and DDE relevant public health concerns.

In a recent integrated systematic review and meta-analysis, $p, p^{\prime}$-DDT and $p, p^{\prime}$-DDE were classified as "presumed" to be obesogenic for humans, based on prospective epidemiological 
observations integrated with experimental evidence of increased rodent adiposity and impaired energy expenditure (5). Numerous studies have suggested that exposure to DDT and/or DDE are additionally associated with several diseases linked to obesity, namely type 2 diabetes (T2D), Alzheimer's disease $(A D)$, and cancer (6-11). However, the mechanism of impairment by DDT or DDE which leads to these diseases remains unresolved. One supporting mechanistic hypothesis is that DDT and DDE are mitotoxicants. Indeed, the role of POPs, including DDT and DDE, in mitochondrial dysfunction and metabolic diseases, such as obesity and T2D has been broadly reviewed (12-14). Furthermore, subtle mitochondrial malfunctions appear to be involved in the pathogenesis of insulin resistance, $\mathrm{T} 2 \mathrm{D}, \mathrm{AD}$, and cancer. However, specific mitochondrial targets of DDT or DDE have not been examined across the existing literature to our knowledge.

The predominant function of mitochondria is the generation of ATP by oxidative phosphorylation (OxPhos), but also includes the generation and detoxification of reactive oxygen species, apoptosis, regulation of calcium, metabolism, self-transportation, and thermogenesis $(15,16)$. Thorough reviews of the methods available to assess mitochondrial dysfunction are available $(15,17)$.

Impaired cellular respiration (18-21) and mitochondrial membrane potential $(18,20)$ have been observed in mammalian mitochondria after exposure to DDT and DDE. DDE also decreased membrane potential, ATP levels, and oxygen consumption rates in human HepG2 cells (22). In this review, we summarize the mechanistic evidence supporting these mitotoxicities by focusing on studies investigating the effect of DDT or DDE on OxPhos, specifically the complexes of the ETC and the efficiency of coupling ATP synthesis to the ETC. We illustrate that both DDT and DDE impair specific complexes of the ETC that contribute to an overall reduction in OxPhos (Table 1 and Figure 1). Although other chemicals may interact with DDT or DDE in targeting OxPhos, exploration of mixture effects is out of the scope of this mini-review.

\section{COMPLEX I}

Complex I (Figure 1) is also known as the NADH dehydrogenase complex or NADH:ubiquinone oxidoreductase. This enzyme complex is responsible for accepting electrons from NADPH and ultimately passing them to the next complex through ubiquinone as protons are pumped across the inner mitochondrial membrane. Systems that are impaired at this complex would have a depression in mitochondrial potential and cellular respiration resulting in one less proton (ATP) produced by the energy transfer of the electron.

Pardini et al. (23) reported a depression of $0-15 \%$ of baseline NADH dehydrogenase activity by $2.5 \mathrm{umol} \mathrm{DDT} / \mathrm{mg}$ of mitochondria from heavy beef heart. However, others did not observe significant defects at Complex I in isolated rat mitochondria at doses above or below that dose (18, 24). Based on the studies reviewed here, there is equivocal evidence implicating a DDT impairment at Complex I. Additional species and conditions are necessary to make a further determination.

Pardini et al. (23) also reported a 5-20\% depression of baseline $\mathrm{NADH}$ dehydrogenase activity by $2.5 \mathrm{umol} / \mathrm{mg}$ of DDE in heavy beef heart mitochondria. Yet Ferreira et al. (20) did not report any changes at Complex I in rat mitochondria exposed to the same range of DDE doses. Without additional evidence it is difficult to reach a conclusion regarding the possible effects of DDE on Complex I; additional studies are necessary to determine the directionality of a DDE effect.

\section{COMPLEX II}

Complex II is contains the enzyme succinate dehydrogenase, also known succinate-CoQ reductase. At this Complex, additional electrons are delivered from the substrate succinate to a quinone pool via flavin adenine dinucleotide (FAD). No protons are transported across the intermembrane space, hence a defect at Complex II would result in a depression of cellular respiration but no change in proton motive force.

Moreno and Madeira (18) reported that Complex II is insensitive to DDT. Conversely, Pardini et al. (23) observed that succinate dehydrogenase enzymatic activity was reduced by 10-20\% after heavy beef mitochondria were treated with DDT and Nishihara and Utsumi (24) observed a 16\% inhibition of succinate dehydrogenase in rat mitochondria after treatment with $50 \mathrm{uM}$ of DDT. Given that Complex II does not contribute to changes in mitochondrial membrane potential, the weak defects by DDT reported here are unlikely to be responsible for the decreased mitochondrial membrane potential observed in mammalian mitochondria exposed to DDT and DDE $(18,20)$, but may cause OxPhos impairment through reduced substrate transfer from Complex I to Complex II (Figure 1).

Pardini et al. (23) also observed enzymatic depressions at Complex II by DDE in heavy beef mitochondria. More compelling yet, while Ferreira et al. (20) reported a depression in respiration and membrane potential in the presence of the Complex II substrate succinate at $10 \mathrm{nmol}$ of DDE in isolated rat mitochondria, this outcome was not observed when Complex III substrates were used to bypass Complex II defects, e.g., ascorbate and the cytochrome $c$ electron donator $N, N, N^{\prime}, N^{\prime}$ Tetramethyl-pphenylenediamine dihydrochloride (TMPD). The full restoration of respiration and membrane potential by ascorbate and TMPD suggests that inhibition of Complex II and inhibition of succinate translocation is the source of DDE depression of mitochondrial respiration. This evidence supports the inference that the inhibitory effects of DDE on Complex II contribute to a limited capacity for ATP production through substrate transfer rather than the proton gradient, to cause an energy imbalance (Figure 1).

\section{COMPLEX III}

Complex III, also referred to as cytochrome $b-c_{1}$, contains at least 11 different polypeptide chains and functions as a dimer. 


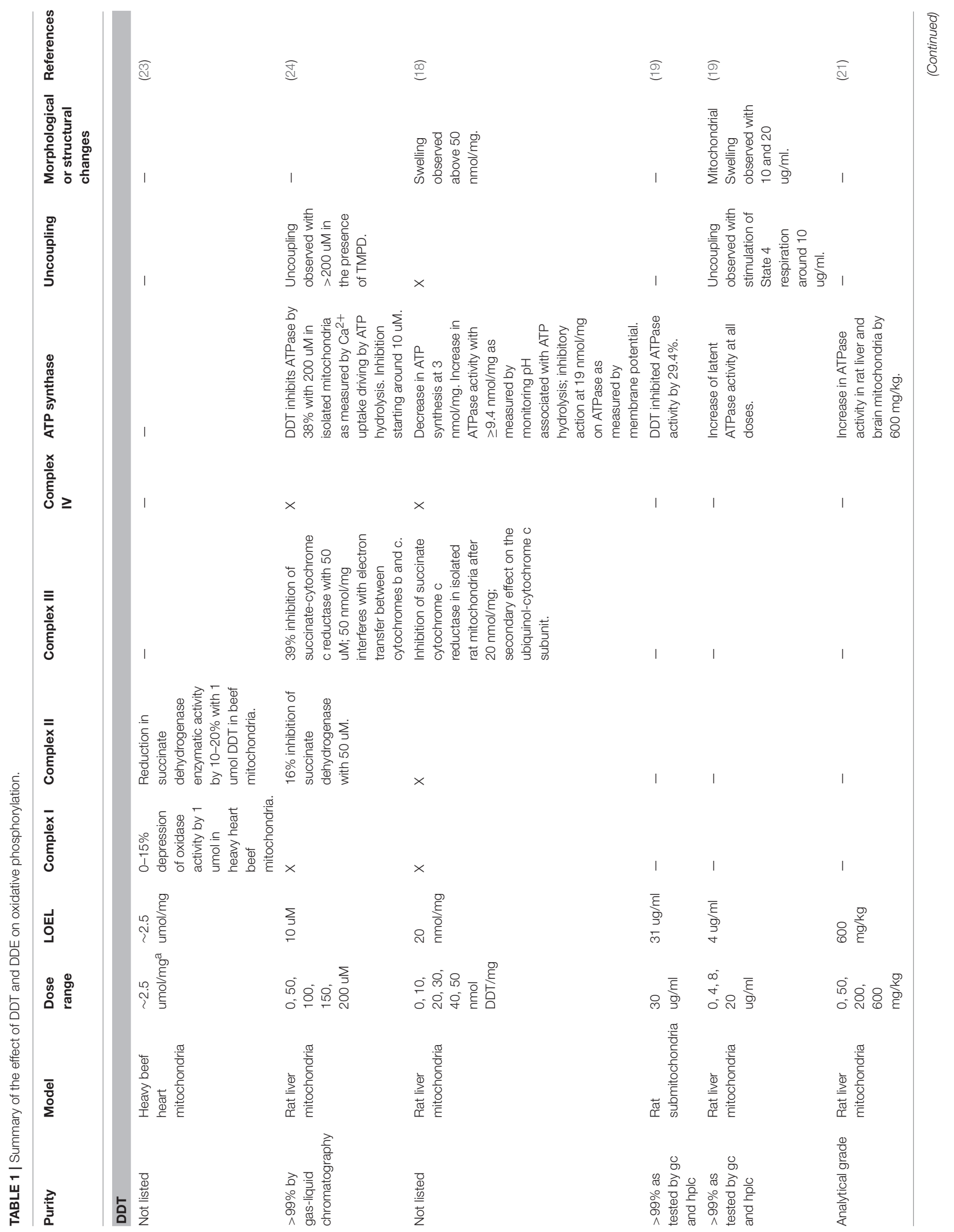




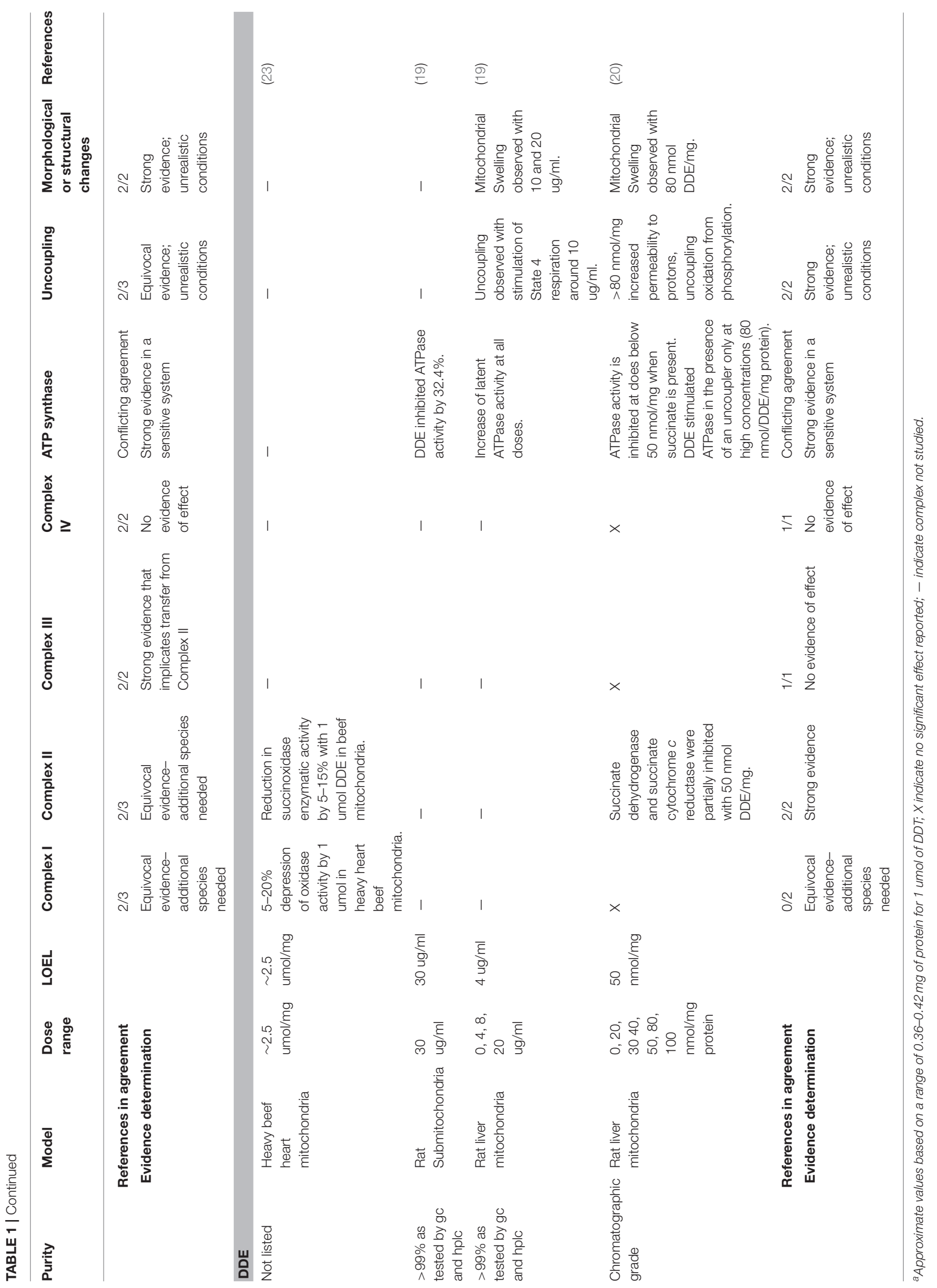




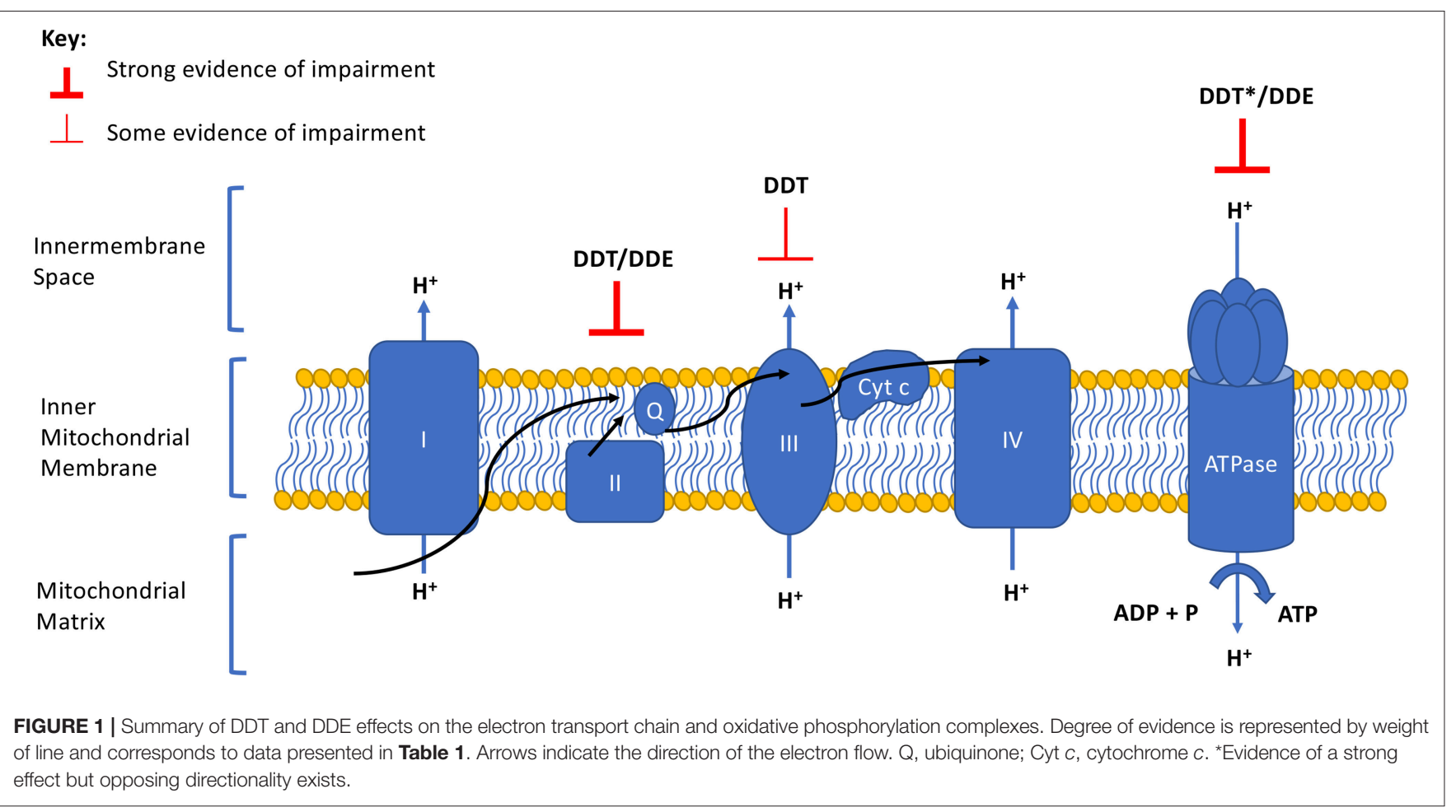

This complex accepts electrons from the substrate ubiquinone and passes them cytochrome $c$, which carries its electron to Complex IV (one electron per cytochrome c); ubiquinolcytochrome-c reductase catalyzes the chemical reaction. At this Complex, protons are transferred to the inner membrane space, contributing to membrane potential.

Nishihara and Utsumi (24) reported that DDT interfered with electron transfer via a 39\% inhibition of succinate-cytochrome c reductase activity after treatment of rat-liver mitochondria with $50 \mathrm{uM}$ DDT. The authors concluded that this defect originated at the electron transfer between cytochrome $b$ and $c$. This effect was only observed when succinate was supplied as the substrate, suggesting that the overall electron transfer defect resulted from a combination of defects at Complex II and III of the ETC. In support of DDT interference with activity between cytochromes $b$ and $c$, Moreno and Madeira (18) observed direct inhibition of the ubiquinol-cytochrome $c$ subunit of isolated rat mitochondria.

Cytochrome $c$ oxidase activity was not impaired when TMPD (serving as a Complex III substrate that bypasses Complex II through its cytochrome $c$ electron donation) was used as the substrate after DDE exposure (20). This observation supports the notion that Complex III machinery and function was intact and working properly after DDE exposure.

\section{COMPLEX IV}

Complex IV, also known as cytochrome $c$ oxidase, is the segment where four electrons are removed from four molecules of cytochrome $c$ and transferred to oxygen to produce two water molecules. Simultaneously, protons are moved from the mitochondrial matrix to the inner membrane thus contributing to the mitochondrial proton gradient.

Both Nishihara and Utsumi (24) and Moreno and Madeira (18) reported that DDT did not affect the cytochrome c oxidase segment of Complex IV.

Ferreira et al. (20) found that Complex IV of the ETC was not affected by DDE.

\section{ATP SYNTHASE (COMPLEX V)}

ATP Synthase (ATPase), often referred to as Complex V, is the final segment of the ETC. It transports a proton into the inner mitochondrial space as energy to increase the proton gradient which fuels the phosphorylation of ADP to ATP.

DDT appears to act on ATPase in every study that has examined it, but the direction of effect is inconsistent (Table 1). On one hand, several authors reported that DDT stimulated ATPase activity in both rat liver and brain mitochondria after DDT treatment $(18,21)$. Similarly, Ohyama et al. (19) reported that DDT stimulated ATPase residing in intact mitochondria yet they also observed an inhibitory effect of DDT on ATPase from sonicated submitochondria particles. These results suggest DDT can inhibit ATPase when ATPase is uncoupled from the ETC. However, other studies of intact mitochondria suggest DDT can inhibit ATPase even when coupled to ETC. For example, Nishihara and Utsumi (24) evaluated isolated rat mitochondria and found weak inhibition of ATPase by DDT starting at $10 \mathrm{uM}$, with maximum inhibition (38\% of control) observed with 200 uM DDT. Further, Moreno and Madeira (18) reported inhibited 
ATPase activity and decreased ATP synthesis in isolated rat mitochondria exposed to low dose DDT.

These differing effects of DDT on ATPase across different methods implemented by Moreno and Madeira may reflect confounding effects by unreported experimental parameters, such as temperature. For example, the motor protein that couples ATP hydrolysis to mechanical rotation was recently characterized by Wantanabe and Noji (25), who found that the rotation of ATPase is highly temperature sensitive. However, despite experimental differences, the consistent perturbation of ATPase activity by DDT among the body of evidence resulting from examination of the effects of DDT on ATPase suggests that ATPase is a target of DDT toxicity and may result in some sort of energy dissipation through Complex V.

Similar to DDT, DDE appears to act on ATPase in most studies reviewed here, but the direction of the DDE effect is inconsistent (Table 1). For example, Ohyama et al. (19) reported a stimulation of "latent" ATPase activity by DDE $(4-20 \mathrm{ug} / \mathrm{ml})$ in isolated rat mitochondria and a DDE $(30 \mathrm{ug} / \mathrm{ml})$ inhibition of ATPase when uncoupled from the ETC in submitochondrial factions. Conversely, Ferreira et al. (20) did not observe any ATPase defects when isolated mitochondria or submitochondrial particles were exposed to 20 or $50 \mathrm{nmol} \mathrm{DDE} / \mathrm{mg}$. Once again, it is reasonable to suggest that ATPase may be a target of DDE toxicity and result in some sort of energy dissipation through the enzyme complex.

\section{ETC UNCOUPLING}

In Complex V, ATPase is responsible for "coupling" the proton gradient of the ETC to ATP synthesis. This process can be uncoupled when uncoupling protein leaks protons back into the inner mitochondrial matrix generating heat rather than producing ATP. Conversely, non-canonical uncoupling can occur in the absence of electron flow and ATPase inhibition, when for other reasons, ATP synthesis cannot take place. These reasons include exposure to uncoupling agents, such as FCCP or CCCP, or to physical force, such as osmotic shock, that dissipates the $\mathrm{pH}$ or membrane potential of the mitochondria (26).

The literature suggests that DDT does not uncouple OxPhos. Moreno and Madeira (18) reported that only large concentrations of DDT caused extensive proton leak. This is consistent with the uncoupled OxPhos by high doses of DDT observed by Ohyama et al. (19) and by Nishihara and Utsumi (24). Given the lipophilicity of DDT, these observations of uncoupled OxPhos following high dose exposure to DDT likely reflect nothing more than non-specific destruction of the mitochondrial membrane.

Similar to DDT experimental outcomes, Ferreira et al. (20) observed partial uncoupling of OxPhos in isolated rat mitochondria at high doses of DDE (>80 nmol/mg protein). Ohyama et al. (19) came to a similar conclusion after reporting uncoupling activity that resulted in stimulation of State 4 respiration. Ferreira and Ohyama suggest that this effect is likely due to disruption of the mitochondrial inner membrane by the high, non-biologically relevant, doses of DDE used.

\section{SUMMARY OF RESULTS AND DISCUSSION OF THE RESEARCH GAPS}

The in vitro studies, primarily in rodent mitochondria, discussed in this review clearly demonstrate the toxic effects of DDT and DDE on Complex II and Complex V of the ETC. Toxicity to Complex II appears to result from substrate disruption. Indeed, given there is no proton transport by Complex II, if DDT and DDE target Complex II, the resulting Complex II perturbation does not explain the reported effects on reduced membrane potential. Instead, we suspect that disruption to ATPase activity by DDT and DDE may contribute to defects associated with mitochondrial respiration and membrane potential. Although inconsistencies in the effects of DDT and DDE on ATPase remain to be resolved, it is important to note that ATPases vary in their sensitivity to DDT depending on temperature (19); this could contribute to different results across systems tested.

Early work presented by Byczkowski (21) suggest mitochondrial uncoupling was the mode of action for DDT mitotoxicity, however it appears this was only the case when DDT or DDE levels exceeded $50 \mathrm{nmol} / \mathrm{mg}$; doses at or above this level often coincided with mitochondrial swelling, an indicator of mitochondrial dysfunction resulting from mitochondrial permeability (27).

Through this review, several mechanistic gaps of DDT and DDE mitotoxicity became apparent. First, given most studies investigated mitochondria from rats and their livers, there is a need for the demonstration of consistency of DDT and DDE mitotoxicity across multiple species and tissues. Given mitochondrial functions vary by cell type and the emerging relationships between DDT, mitochondrial-dense brown adipose tissue, and obesity [e.g., (28)], this is a tissue in need of characterization. Additionally, given the evidence supporting a role of substrate perturbation in Complex II toxicity caused by DDT and DDE, whole cell and/or ETC substrate studies should be conducted. Lastly, the direction of DDT and DDE effects on ATPase function should be resolved at doses more relevant to the human condition. In the meta-analysis of prospective human studies associating DDTs with obesity, Cano-Sancho et al. (5) found internal concentrations of DDT and DDE to be between 0.001 and $10 \mathrm{ng}$ DDTs $/ \mathrm{mL}$. Based on lipid weight conversion as described by Cano-Sancho et al. (5), these obesogenic levels correspond to $\sim 0.001-30 \mathrm{nM}$ of DDT or DDE for in vitro dosing. We further suggest that temperature and perhaps pressure be systematically controlled in this endeavor to resolve discrepancies in the DDT and DDE effect at ATPase.

\section{IMPLICATIONS FOR DISEASE ETIOLOGY}

An increasing number of studies suggest a strong role for DDT and/or DDE in the etiology of human disease including obesity, $\mathrm{T} 2 \mathrm{D}, \mathrm{AD}$, and cancer. Based on this review, mitotoxic effects targeting OxPhos appear to be a likely consequence of DDT or DDE exposure which could contribute to the pathogenesis of such diseases. 
Obesity is the result of disturbances in energy balance. Rates of obesity are rising in humans and other animals, including primates and rodents serving as experimental controls, feral rodents, and domestic dogs and cats (29) suggesting an etiology beyond overeating and/or inactivity. One source of disturbance in energy metabolism is mitochondrial dysfunction, given the organelle's central role in ATP production and energy expenditure including consequences on lipid and glucose metabolism (30-32). Moreover, the term obesogen has been coined for toxicants that cause such disturbances. Based on meta-analysis of human prospective studies and bioassays, DDT and DDE have been presumed to be obseogens (5). Developmental DDT exposure increased rodent obesity in subsequent generations, where it impaired thermogenesis and decreased energy expenditure while reducing RNA coding for mitochondrial control of thermogenesis and energy expenditure in mice $(28,33)$.

Similar to obesity trends, the prevalence of T2D has risen dramatically in countries of all incomes (34). T2D is characterized by defects in both insulin action and insulin secretion with emerging evidence that mitochondria dysfunction causes both (35). For example Petersen et al. (36) used $13^{\mathrm{C}}$ and $31^{\mathrm{P}}$ magnetic resonance spectroscopy to demonstrate that insulin resistance could be accompanied by a reduction in mitochondrial oxidative activity and mitochondrial ATP synthesis (36). This mechanism is consistent with work in rodents that demonstrated impaired insulin secretion and action after exposure to DDT $(28,37)$, and in humans, DDE is associated with T2D (9). The elevated T2D risk observed could arise from decreased mitochondrial membrane potential, ATP levels, and oxygen consumption rates in insulin responsive hepatocytes after DDE exposure (22).

$\mathrm{AD}$ is the sixth leading cause of death in the U.S. (38) with poorly understood causes. Its link to mitochondrial activity has recently been explored in a mouse model for familial AD where an age-dependent decrease in mitochondrial complex-II activity starting at 9 months was observed (39). In a separate study of human hippocampal tissues from non-AD controls and $\mathrm{AD}$ cases, genes involved in OxPhos were significantly down regulated in subjects with $\mathrm{AD}$ including genes involved in both complexes II and V (40). Indeed mitochondrial dysfunction may cause energy failures in neurons to induce synaptic dysfunction underlying cognitive impairment (40). The dysregulation of Complexes II and V by DDT and DDE in the pathogenesis of

\section{REFERENCES}

1. World Health Organization. The Use of DDT in Malaria Vector Control. WHO Position Statement. Geneva: World Health Organization (2011).

2. Federal Drug Administration (FDA). CPG Sec. 575.100 Pesticide Residues in Food and Feed-Enforcement Criteria. Silver Spring, MD (2018).

3. Sanz-Gallardo MI, Guallar E, Van't Veer P, Longnecker MP, Strain JJ, Martin $\mathrm{BC}$, et al. Determinants of $\mathrm{p}, \mathrm{p}^{\prime}$-dichlorodiphenyldichloroethane (DDE) concentration in adipose tissue in women from five European cities. Arch Environ Health Int J. (1999) 54:277-83. doi: 10.1080/00039899909602486

4. Rivero-Rodriguez L, Borja-Aburto VH, Santos-Burgoa C, Waliszewskiy S, Rios C, Cruz V. Exposure assessment for workers applying DDT to control
$\mathrm{AD}$ is consistent with two molecular epidemiology studies which found an association between elevated DDE serum levels and AD $(8,41)$.

In many regards, cancer is a disease of mitochondrial dysfunction characterized by a metabolic shift to anaerobic conditions including mutations in genes encoding mitochondrial proteins (42). DDT has been listed by the California Environmental Protection Agency as an agent causing cancer (43) and classified as "probably carcinogenic to humans" (Group $2 \mathrm{~A})$ by the IARC $(10,11)$, although little has been reported on the mode of DDT's carcinogenic action. Defects in succinate dehydrogenase (complex II), among other mitochondrial enzymes, are associated with both familial and sporadic forms of cancer $(42,44)$ which is consistent with the effects of DDT and DDE on Complex II reviewed here. DDT and DDE mitotoxicity could hence contribute to at least two key characteristics of cancer (45): (1) through interruption of mitochondrial OxPhos (KC: "induces oxidative stress") and (2) affecting cellular nutrient supply by altering ATP synthesis (KC: "alters cell proliferation, cell death, or nutrient supply").

\section{CONCLUSION}

In summary, there is strong evidence for OxPhos impairment at Complexes II and V by DDT and DDE which in turn could cause or contribute to the etiology of diseases, such as obesity, T2D, AD, and cancer. Future work should consider the experimental details mentioned in this review when investigating the role of DDT and DDE as Complex II and Complex $\mathrm{V}$ mitotoxicants as a potential mechanistic causes of these diseases and ideally, use that knowledge to develop therapeutic treatments.

\section{AUTHOR CONTRIBUTIONS}

ML conceived of the topic, directed and led the literature search, interpreted data, revised manuscript. SE coled the literature search, interpreted data, drafted, and revised manuscript.

\section{FUNDING}

This research was funded by NIH R01ES024946. The NIH had no direct role of the manuscript content. malaria in Veracruz, Mexico. Environ Health Perspect. (1997) 105:98-101. doi: $10.1289 /$ ehp. 9710598

5. Cano-Sancho G, Salmon AG, La Merrill MA. Association between exposure to $\mathrm{p}, \mathrm{p}^{\prime}-\mathrm{DDT}$ and its metabolite $\mathrm{p}, \mathrm{p}^{\prime}-\mathrm{DDE}$ with obesity: integrated systematic review and meta-analysis. Environ Health Perspect. (2017) 125:096002. doi: 10.1289/EHP527

6. Cox S, Niskar AS, Narayan KM, Marcus M. Prevalence of self-reported diabetes and exposure to organochlorine pesticides among Mexican Americans: hispanic health and nutrition examination survey, 1982-1984. Environ Health Perspect. (2007) 115:1747-52. doi: 10.1289/ehp.10258

7. Lee DH, Steffes MW, Sjödin A, Jones RS, Needham LL, Jacobs Jr. DR. Low dose organochlorine pesticides and polychlorinated biphenyls predict obesity, 
dyslipidemia, and insulin resistance among people free of diabetes. PLOS ONE. (2011) 6:e15977. doi: 10.1371/journal.pone.0015977

8. Richardson JR, Roy A, Shalat SL, von Stein RT, Hossain MM, Buckley B, et al. Elevated serum pesticide levels and risk of Alzheimer disease. JAMA Neurol. (2014) 71:284-90. doi: 10.1001/jamaneurol.2013.6030

9. Evangelou E, Ntritsos G, Chondrogiorgi M, Kavvoura FK, Hernández AF, Ntzani EE, et al. Exposure to pesticides and diabetes: a systematic review and meta-analysis. Environ Int. (2016) 91:60-8. doi: 10.1016/j.envint.2016.02.013

10. Loomis D, Guyton K, Grosse Y, El Ghissasi F, Bouvard V, Benbrahim-Tallaa $\mathrm{L}$, et al. Carcinogenicity of lindane, DDT, and 2,4-dichlorophenoxyacetic acid. Lancet Oncol. (2015) 16:891-92. doi: 10.1016/S1470-2045(15)00081-9

11. International Agency for Research on Cancer. IARC Monographs on the Evaluation of Carcinogenic Risk to Humans. Vol. 113. Lyon (2018).

12. Lim S, Cho YM, Park KS, Lee HK. Persistent organic pollutants, mitochondrial dysfunction and metabolic syndrome. Ann N Y Acad Sci. (2010) 1201:166-76. doi: $10.1111 / j .1749-6632.2010 .05622 . x$

13. Kim KS, Lee YM, Kim SG, Lee IK, Lee HJ, Kim J, et al. Associations of organochlorine pesticides and polychlorinated biphenyls in visceral vs. subcutaneous adipose tissue with type 2 diabetes and insulin resistance. Chemosphere. (2014) 94:151-7. doi: 10.1016/j.chemosphere.2013.09.066

14. Lee DH, Porta M, Jacobs DR, Vandenberg LN. Chlorinated persistent organic pollutants, obesity, and type 2 diabetes. Endocr Rev. (2014) 35:557-601. doi: $10.1210 /$ er.2013-1084

15. Brand MD, Nicholls DG. Assessing mitochondrial dysfunction in cells. Biochem J. (2011) 435:291-312. doi: 10.1042/BJ20110162

16. Cannon B, Nedergaard J. Brown adipose tissue: function and physiological significance. Physiol Rev. (2004) 84:277-359. doi: 10.1152/physrev.00015.2003

17. Meyer JN, Hartman JH, Mello DF. Mitochondrial toxicity. Toxi Sci. (2018) 162:15-23. doi: 10.1093/toxsci/kfy008

18. Moreno AJ, Madeira VM. Mitochondrial bioenergetics as affected by DDT. Biochim Biophys Acta. (1991) 1060:166-74. doi: 10.1016/S0005-2728(09)91004-0

19. Ohyama T, Takahasi T, Ogawa H. Effects of dichlorodiphenyltrichloroethane and its analogues on rat liver mitochondria. Biochem Pharmacol. (1982) 31:397-404. doi: 10.1016/0006-2952(82)90188-5

20. Ferreira FM, Madeira VM, Moreno AJ. Interactions of 2,2bis(p-chlorophenyl)-1,1-dichloroethylene with mitochondrial oxidative phosphorylation. Biochem Pharmacol. (1997) 53:299-308. doi: 10.1016/S0006-2952(96)00689-2

21. Byczkowski JZ. The mode of action of $\mathrm{p}, \mathrm{p}^{\prime}-\mathrm{DDT}$ on mammalian mitochondria. Toxicology. (1976) 6:309-14. doi: 10.1016/0300-483X(76) 90034-2

22. Liu Q, Wang $\mathrm{Q}, \mathrm{Xu} \mathrm{C}$, Shao W, Zhang $\mathrm{C}$, Liu H, et al. Organochloride pesticides impaired mitochondrial function in hepatocytes and aggravated disorders of fatty acid metabolism. Sci Rep. (2017) 7:46339. doi: $10.1038 /$ srep46339

23. Pardini RS, Heidker JC, Baker TA, Payne B. Toxicology of various pesticides and their decomposition products on mitochondrial electron transport. Arch Environ Contam Toxicol. (1980) 9:87-97. doi: 10.1007/BF01055502

24. Nishihara Y, Utsumi K. Effects of 1,1,1-trichloro-2,2-bis-(pchlorophenyl)ethane (DDT) on ATPase-linked functions in isolated rat-liver mitochondria. Food Chem Toxicol. (1985) 23:599-602.25

25. Wantanabe R, Noji H. Characterization of the temperature-sensitive reaction of $\mathrm{F}_{1}$-ATPase by suing single-molecule manipulation. Sci Rep. (2014) 4:4962. doi: 10.1038/srep04962

26. Terada H. Uncouplers of oxidative phosphorylation. Environ Health Perspect. (1990) 87:213-8. doi: 10.1289/ehp.9087213

27. Gerencser AA, Doczi J, Töröcsik B, Bossy-Wetzel E, Adam-Vizi V. Mitochondrial swelling measurement in situ by optimized spatial filtering: astrocyte-neuron differences. Biophys J. (2008) 95:2583-98. doi: 10.1529/biophysj.107.118620

28. La Merrill M, Karey E, Moshier E, Lindtner C, La Frano MR, Newman JW, et al. Perinatal exposure of mice to the pesticide DDT impairs energy expenditure and metabolism in adult female offspring. PLOS ONE. (2014) 9:e103337. doi: 10.1371/journal.pone.0103337

29. Klimentidis YC, Beasley TM, Lin HY, Murati G, Glass GE, Guyton $\mathrm{M}$, et al. Canaries in the coal mine: a cross-species analysis of the plurality of obesity epidemics. Proc R Soc B Biol Sci. (2010) 278:1626-32. doi: $10.1098 / \mathrm{rspb} .2010 .1890$

30. Namwanje M, Bournat JC, Brown CW. Isolation and manipulation of adipogenic cells to assess TGF- $\beta$ superfamily functions. In: TGF- $\beta$ Signaling. New York, NY: Humana Press (2016). p. 205-17.

31. Liesa M, Shirihai OS. Mitochondrial dynamics in the regulation of nutrient utilization and energy expenditure. Cell Metab. (2013) 17:491-506. doi: 10.1016/j.cmet.2013.03.002

32. Bhatti JS, Bhatti GK, Reddy PH. Mitochondrial dysfunction and oxidative stress in metabolic disorders-a step towards mitochondria based therapeutic strategies. Biochim Biophys Acta. (2016) 1863:1066-77. doi: 10.1016/j.bbadis.2016.11.010

33. Skinner MK, Manikkam M, Tracey R, Guerrero-Bosagna C, Haque M, Nilsson EE. Ancestral dichlorodiphenyltrichloroethane (DDT) exposure promotes epigenetic transgenerational inheritance of obesity. BMC Med. (2013) 11:228. doi: 10.1186/1741-7015-11-228

34. World Health Organization. Obesity and overweight: Fact Sheet. Geneva: World Health Organization (2018).

35. Lowell BB, Shulman GI. Mitochondrial dysfunction and type 2 diabetes. Science. (2005) 307:384-7. doi: 10.1126/science.1104343

36. Petersen KF, Befroy D, Dufour S, Dziura J, Ariyan C, Rothman DL, et al. Mitochondrial dysfunction in the elderly: possible role in insulin resistance. Science. (2003) 300:1140-2. doi: 10.1126/science. 1082889

37. Yau ET, Mennear JH. The inhibitoty effect of DDT on insulin secretion in mice. Toxicol Appl Pharmacol. (1977) 39:81-8. doi: 10.1016/0041-008X(77)90179-X

38. NIH, National Institute on Aging. Alzheimer's Disease and Related Demntias. (2016). Available online at: https://www.nia.nih.gov/health/ alzheimers-disease-fact-sheet

39. Emmerzaal TL, Rodenburg RJ, Tanila H, Verweij V, Kiliaan AJ, Kozicz T. Age-dependent decrease of mitochondrial complex II activity in a familial mouse model for Alzheimer's disease. J Alzheimers Dis. (2018) 66:75-82. doi: 10.3233/JAD-180337

40. Mastroeni D, Khdour OM, Delvaux E, Nolz J, Olsen G, Berchtold N, et al. Nuclear but not mitochondrial-encoded oxidative phosphorylation genes are altered in aging, mild cognitive impairment, and Alzheimer's disease. Alzheimers Dement. (2017) 13:510-9. doi: 10.1016/j.jalz.2016. 09.003

41. Bible E. High serum levels of the pesticide metabolite DDE-a potential environmental risk factor for Alzheimer disease. Nat Rev Neurol. (2014) 10:125-6. doi: 10.1038/nrneurol.2014.25

42. Piruat JI, Millán-Uclés Á. Genetically modeled mice with mutations in mitochondrial metabolic enzymes for the study of cancer. Front Oncol. (2014) 4:200. doi: 10.3389/fonc. 2014.00200

43. Cal/EPA-OEHHA. California Environmental Protection Agency-Office of Environmental Health Hazard Assessment, Safe Drinking Water and Toxic Enforcement Act of 1986. Chemicals Known to the State to Cause Cancer or Reproductive Toxicity. Sacramento, CA (1987).

44. Hsu CC, Tseng LM, Lee HC. Role of mitochondrial dysfunction in cancer progression. Exp Biol Med. (2016) 241:1281-95. doi: $10.1177 / 1535370216641787$

45. Smith MT, Guyton KZ, Gibbons CF, Fritz JM, Portier CJ, Rusyn I, et al. Key characteristics of carcinogens as a basis for organizing data on mechanisms of carcinogenesis. Environ Health Perspect. (2015) 124:713-21. doi: $10.1289 /$ ehp. 1509912

Conflict of Interest Statement: The authors declare that the research was conducted in the absence of any commercial or financial relationships that could be construed as a potential conflict of interest.

Copyright $\odot 2019$ Elmore and La Merrill. This is an open-access article distributed under the terms of the Creative Commons Attribution License (CC BY). The use, distribution or reproduction in other forums is permitted, provided the original author(s) and the copyright owner(s) are credited and that the original publication in this journal is cited, in accordance with accepted academic practice. No use, distribution or reproduction is permitted which does not comply with these terms. 